\title{
Safety and efficacy of conversion to once-daily tacrolimus from twice-daily tacrolimus in pediatric liver transplant recipients
}

\author{
${ }^{1}$ Department of Surgery, Samsung Medical Center, Seoul, Korea \\ ${ }^{2}$ Division of Pediatric Surgery, Department of Surgery, Samsung Medical Center, Seoul, Korea \\ ${ }^{3}$ Division of Transplantation, Department of Surgery, Samsung Medical Center, Seoul, Korea \\ ${ }^{4}$ Division of Transplantation, Department of Surgery, Vinmec Healthcare System, Hanoi, Vietnam
}

Sung Hyo An ${ }^{1}$, Sanghoon Lee ${ }^{2}$, Jinsoo Rhu ${ }^{3}$, Jong Man Kim ${ }^{3}$, Gyu Seong Choi ${ }^{3}$, Jae-Won Joh ${ }^{3}$, Suk-Koo Lee ${ }^{4}$

Background: Nonadherence of immunosuppression is the most common cause of late acute rejection in pediatric liver recipients. Simpler dosing regimens promote better compliance behavior, especially in pediatric patients.

Methods: We screened 182 pediatric liver transplant (LT) recipients converted from twice daily tacrolimus (TD-TAC, Prograf) to once daily tacrolimus (OD-TAC, Advagraf) between February 2011 and September 2019. Tacrolimus trough levels, doses of tacrolimus, and liver function tests (LFT) were recorded in 3 months intervals 18 months before and after conversion.

Results: One hundred and seventy-nine patients were converted to OD-TAC and followed for 18 months. One hundred and fifty-four out of 179 patients (86.0\%) converted to OD-TAC and were uneventful during follow-up. Twenty-one patients had LFT elevation above $80 \mathrm{U} / \mathrm{L}$. These patients' LFT normalized after OD-TAC dose adjustment $(n=12)$ or switching back to TD-TAC $(n=9)$. Four patients had biopsy-proven acute rejection within 6 months after conversion, and all of which were successfully treated with steroid pulse. Currently, 166 out of 179 patients (92.7\%) remain on OD-TAC and 13 patients (7.3\%) were switched back to TD-TAC. Mean tacrolimus trough level significantly decreased at 3 months $(3.14 \pm 1.9 \mathrm{ng} / \mathrm{mL})$ following conversion compared to pre-conversion level $(3.69 \pm 1.98 \mathrm{ng} / \mathrm{mL})$. Mean tacrolimus trough levels remained unchanged from 3 to 12 months following conversion. Mean daily tacrolimus dosage was increased from pre-conversion dosage $(1.60 \pm 1.29 \mathrm{mg})$ to initial conversion dose $(1.76 \pm 1.31$ $\mathrm{mg})$ and once again at 3 months $(1.80 \pm 1.23 \mathrm{mg})$ following conversion. Tacrolimus dosage remained unchanged from 3 to 12 months. Percent coefficient of variation of tacrolimus trough levels was significantly decreased from $32.5 \pm 16.4$ to $27.5 \pm 15.6 \mathrm{ng} /$ $\mathrm{mL}$ after conversion to OD-TAC, reflecting the decrease in variation of tacrolimus trough levels following conversion.

Conclusions: Conversion to OD-TAC in pediatric LT recipients with stable graft function is safe and effective.

Corresponding author: Sanghoon Lee

E-mail: sanghoone.lee@samsung.com

(c) The Korean Society for Transplantation

This is an Open Access article distributed under the terms of the Creative Commons Attribution Non-Commercial License (http://creativecommons.org/licenses/by-nc/4.0/) which permits unrestricted non-commercial use, distribution, and reproduction in any medium, provided the original work is properly cited. 